\title{
JD10
}

\section{Evolution in Galaxy Clusters: A Multiwavelength Approach}

Chairpersons: L. Feretti and R.W. Hunstead

Editors: R.W. Hunstead (Chief-Editor), L. Feretti, B. Gibson and P. Nulsen 


\title{
Evolution in Galaxy Clusters: A Multi-Wavelength Approach
}

\author{
Joint Discussion 10, 17-18 July 2003
}

\section{Organizing Division: $\mathrm{X}$}

\section{Participating Divisions: VIII and XI}

SOC members: R. Hunstead (Australia, chair), L. Feretti (Italy, co-chair), R. Carlberg (Canada), W. Couch (Australia), B. Gibson (Australia), G. Kauffmann (Germany), L. Lubin (USA), G. Miley (The Netherlands), P. Nulsen (Australia), T. Ohashi (Japan), P. Thomas (UK)

As the largest virialized systems in the universe, clusters of galaxies are astrophysical laboratories for studying a wide variety of phenomena. These range from the properties of individual galaxies, to the intra-cluster medium through which they move and the violent events that accompany cluster mergers. Observational data span the entire electromagnetic spectrum from X-ray through UV to optical, infrared and radio. Each wavelength region reveals a different facet of the underlying astrophysics that governs the gas and galaxies.

In this context, we felt it was timely to schedule a Joint Discussion about clusters and their evolution within the IAU General Assembly in Sydney. The multi-wavelength theme emphasized the above mentioned synergies, and linked the exciting new observational data from ground-based and space observatories to the most recent analytical and numerical simulations.

Joint Discussion 10 focused on some recent highlights of cluster research: (i) cluster formation, with discussion of the optical and X-ray data, substructure, mergers and accretion; (ii) cluster galaxies, with emphasis on the star-formation histories and radio galaxy populations; and (iii) the intra-cluster medium, with the recent results on the gas cavities, cooling flows, non-thermal processes and the interaction between thermal and relativistic plasma. Since it was only a 1.5-day meeting, the number of oral contributions was limited to 10 invited and 12 contributed talks. Other contributions were presented as Posters, and a short presentation of each poster was given during the oral session.

In the following sections we present an abbreviated version of the program, followed by the texts of 8 of the 10 invited talks, the abstracts of the contributed talks, and the list of posters.

We thank all the participants for their contributions to a productive and successful meeting.

Dick Hunstead Luigina Feretti 